\title{
openheart The relationship between oxidised LDL, endothelial progenitor cells and coronary endothelial function in patients with CHD
}

\author{
Jonathan Watt, ${ }^{1}$ Simon Kennedy, ${ }^{2}$ Nadeem Ahmed, ${ }^{2}$ James Hayhurst, ${ }^{2}$
} John D McClure, Colin Berry, ${ }^{1}$ Roger M Wadsworth, ${ }^{3}$ Keith G Oldroyd ${ }^{1}$

To cite: Watt J, Kennedy S, Ahmed N, et al. The relationship between oxidised LDL, endothelial progenitor cells and coronary endothelial function in patients with CHD. Open Heart 2016;3: e000342. doi:10.1136/ openhrt-2015-000342

Received 29 September 2015 Revised 18 December 2015 Accepted 20 December 2015

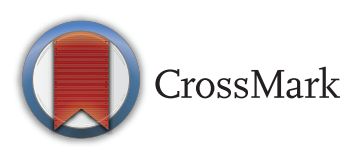

${ }^{1}$ West of Scotland Regional Heart \& Lung Centre, Golden Jubilee National Hospital, Glasgow, UK

${ }^{2}$ Institute of Cardiovascular and Medical Sciences, University of Glasgow, Glasgow, UK

${ }^{3}$ Strathclyde Institute of Pharmacy and Biomedical Sciences, University of Strathclyde, Glasgow, UK

Correspondence to Dr Simon Kennedy; simon.kennedy@glasgow.ac. uk

\section{ABSTRACT}

Objective: The balance between coronary endothelial dysfunction and repair is influenced by many protective and deleterious factors circulating in the blood. We studied the relationship between oxidised low-density lipoprotein (oxLDL), circulating endothelial progenitor cells (EPCs) and coronary endothelial function in patients with stable coronary heart disease (CHD). Methods: 33 patients with stable CHD were studied. Plasma oxLDL was measured using ELISA, coronary endothelial function was assessed using intracoronary acetylcholine infusion and EPCs were quantified using flow cytometry for $\mathrm{CD}^{+} 4^{+} / \mathrm{KDR}^{+}$cells.

Results: Plasma oxLDL correlated positively with the number of EPCs in the blood $(r=0.46, p=0.02)$. There was a positive correlation between the number of circulating EPCs and coronary endothelial function $(r=0.42, p=0.04)$. There was no significant correlation between oxLDL and coronary endothelial function.

Conclusions: Plasma levels of oxLDL are associated with increased circulating EPCs in the blood of patients with $\mathrm{CHD}$, which may reflect a host-repair response to endothelial injury. Patients with stable CHD had a high prevalence of coronary endothelial dysfunction, which was associated with lower numbers of circulating EPCs, suggesting a mechanistic link between endothelial dysfunction and the pathogenesis of atherosclerosis.

\section{INTRODUCTION}

Oxidised low-density lipoprotein (oxLDL) injures the vascular endothelium, a key step in the pathogenesis of atherosclerosis. ${ }^{1}$ An important mechanism of endothelial repair involves the mobilisation and homing of bone marrow-derived endothelial progenitor cells (EPCs) to sites of injury, repopulating the artery with functional endothelial cells (ECs). Circulating levels of EPCs in blood are increased following many forms of endothelial injury including ischaemia in experimental animals ${ }^{2}$ and myocardial infarction in humans, ${ }^{34}$ but there are conflicting data on

\section{KEY QUESTIONS}

What is already known about this subject?

- Endothelial progenitor cells (EPCs) are crucial mediators of vascular repair following endothelial injury and the number of circulating EPCs is influenced by many factors, including myocardial ischaemia and levels of proinflammatory cytokines.

What does this study add?

- We found the number of circulating EPCs was correlated with plasma oxidised low-density lipoprotein (oxLDL) in patients with stable coronary heart disease, most of whom were taking statins and other cardioprotective medications. The number of circulating EPCs also correlated with a measure of coronary endothelial function.

How might this impact on clinical practice?

- Our study suggests a host-repair response to oxLDL, demonstrated by an increase in circulating EPCs. Elevated levels of EPCs have the potential to improve endothelial function. Our data highlight the potential role of targeted measures to promote endothelial function which, in turn, may improve cardiovascular health.

whether circulating EPC levels correspond to the severity of coronary heart disease (CHD). ${ }^{5}{ }^{6}$ EPCs are more resistant than mature ECs to the toxic effects of oxidative stress due to greater expression of potent antioxidant enzymes ${ }^{7}$ that allow EPCs to proliferate and differentiate in areas of increased oxidative stress, such as ischaemic tissues. EPC mobilisation is known to be triggered by several proinflammatory cytokines and growth factors including granulocyte macrophage-colony stimulating factor, stromal cell-derived factor-1, matrix metalloproteinases, vascular endothelial growth factor (VEGF) and erythropoetin. ${ }^{8}$ In laboratory studies, oxLDL is toxic to cultured 
EPCs, ${ }^{9-12}$ however, the in vivo relationship between plasma oxLDL and the number of circulating EPCs in the blood is not known. Lowering oxLDL in humans pharmacologically has a heterogeneous effect on endothelial dysfunction, with most coronary segments showing enhancement of dilation to acetylcholine (ACh), but other segments showing a reduction in dilation. ${ }^{13}$ We hypothesised that elevated plasma oxLDL in patients with stable CHD would result in an increase in circulating EPCs and that this may have a positive effect on endothelial function. The aim of this study was therefore to investigate the relationship between plasma oxLDL and EPCs in patients with stable CHD and whether circulating EPCs influence coronary endothelial function. To our knowledge, this is the first study to investigate this relationship and our results provide evidence that elevated oxidative stress may stimulate mechanisms involved in endothelial repair, thereby exerting an effect on endothelial function.

\section{METHODS}

\section{Study population}

This cross-sectional observational study was approved by the West Glasgow ethics committee (05/S0709/ 138-Coronary stent deployment, oxidative stress, endothelial regeneration and risk of thrombosis) and the investigation conformed with the principles outlined in the Declaration of Helsinki. All individual data were collected in a blinded fashion. All participants were provided with a Patient Information Sheet and gave informed written consent. To be included in the study, patients had to be over age 18 and scheduled to undergo elective percutaneous coronary intervention (PCI) to treat stable CHD. All patients had at least one major epicardial coronary artery suitable for endothelial function testing $(<30 \%$ stenosis and at least $2.5 \mathrm{~mm}$ in diameter). Patients were excluded if there was a history of myocardial infarction (MI) within 3 months or if they were unable to give informed consent. One hour before PCI, venous blood was removed for immediate assay of circulating EPCs and plasma was stored at $-70^{\circ} \mathrm{C}$ for subsequent oxLDL assay. Patients then underwent routine cardiac catheterisation as planned with the additional component of invasive coronary endothelial function testing prior to PCI.

\section{OxLDL assay}

OxLDL was detected by the commercial Mercodia solid two-site ELISA (Diagenics, Bletchley, UK). In this assay, two monoclonal antibodies were directed against separate antigenic determinants on the oxidised apolipoprotein B molecule. Samples were snap-thawed at $37^{\circ} \mathrm{C}$ for $3 \mathrm{~min}$. All samples were run in duplicate. The average coefficient of variation of the duplicates was $0.6 \%$. The lowest sample value was $36 \mathrm{IU}$, which was above the lowest standard (10 IU). All samples were therefore within the sensitivity of the assay.

\section{EPC assay}

EPC sample preparation involved $1 \mathrm{~mL}$ of EDTA anticoagulated blood added to $100 \mu \mathrm{L}$ of Fc receptor (FcR) blocking reagent (Miltenyi Biotec) and incubated for $20 \mathrm{~min}$ at room temperature. FCR blocking reagent stopped the non-specific binding of $\operatorname{IgG}$, reducing the background noise in the sample. Five tubes were then prepared, numbered $1-5$, with $20 \mu \mathrm{L}$ of anti-VEGF receptor-2 APC added to tubes $1,3,4,5$ and $20 \mu \mathrm{L}$ IgG1-APC was added to tube 2. One hundred microlitres of the blocked blood, containing the FcR blocking reagent, was then added to each of the tubes (1-5). All tubes were incubated for a further $30 \mathrm{~min}$ on ice and protected from light. Twenty microlitres of 7 amino-actinomycin D visibility dye was added to each tube for the exclusion of non-viable cells. Twenty microlitres of CD45-FITC/CD34-PE antibody combination was then added to tubes 2, 3, 4, 5, with $20 \mu \mathrm{L}$ of CD45-FITC/CTL-PE added to tube 1 . These tubes were incubated at room temperature for $20 \mathrm{~min}$ and protected from light. Essentially, tube 1 acted as a control for CD34 using CD45-FITC/CTL-PE and tube 2 as a control for KDR, by adding CD45-FITC/CD34-PE. Diluted lysis buffer was made up by adding $400 \mu \mathrm{L} 10 \times$ buffer to $3.6 \mathrm{~mL}$ of water. After $20 \mathrm{~min}$ incubation, $500 \mu \mathrm{L}$ of lysis buffer was added to each tube before a further $10 \mathrm{~min}$ of incubation in the dark. Finally, $100 \mu \mathrm{L}$ of a fluorosphere stem count reagent was added to each tube to prepare the sample for fluorescence-activated cell sorting (FACS) acquisition.

EPCs were quantified by flow cytometry using FACS analysis to look for markers CD34 and KDR, thought to define EPCs. ${ }^{14}$ Data were analysed using Cellquest Pro Software (BD) which involved the use of analytical gates to count the total number and subsets of circulating cells. Samples were run until 250000 events were counted or $20 \mathrm{~min}$ had elapsed. Data were then stored for future analysis where EPCs were quantified as ((the number of $\left.\mathrm{CD} 34^{+} \mathrm{KDR}^{+}\right)$events $) \times($concentration of fluorospheres))/(total number of fluorospheres) per $\mu \mathrm{L}$ of whole blood.

\section{Coronary endothelial function testing}

All vasodilator medications were withheld for $24 \mathrm{~h}$ prior to testing. Coronary endothelial function was measured by the standard method of assessing the change in luminal diameter, using quantitative coronary angiography (QCA), in response to an intracoronary infusion of the endothelium-dependent vasodilator, ACh. In healthy arteries, ACh causes release of nitric oxide (NO) from ECs, leading to vasodilation. In endothelial dysfunction, the $\mathrm{NO}$ response is blunted and the direct muscarinic smooth muscle response to ACh predominates, causing paradoxical vasoconstriction. Following baseline coronary angiography, the optimal angiographic projection for the study artery was selected, avoiding overlapping side-branches. A 3 French infusion catheter (Cook Medical, Limerick, Ireland) was placed into the proximal portion of the 
artery to be studied via a standard 6 French guiding catheter. After intracoronary infusion of $0.9 \%$ saline as a control, endothelium-dependent vasomotion was assessed by serial infusions of ACh (Miochol-E, Novartis) in the following order: $10^{-6}, 10^{-5}$ and $10^{-4} \mathrm{M}$ at a flow rate of $2 \mathrm{~mL} / \mathrm{min}$ for $2 \mathrm{~min}$. Assuming a mean coronary artery blood flow of $50 \mathrm{~mL} / \mathrm{min}$, the final blood concentration for each ACh infusion was $4 \times 10^{-8}, 4 \times 10^{-7}$ and $4 \times 10^{-6} \mathrm{M}$, which was appropriate for the assessment of vasomotor responses (recommended range $10^{-8}$ to $10^{-5} \mathrm{M}$ ) ${ }^{15}$ At the end of each infusion, coronary angiography was performed with contrast medium (Omnipaque, GE Healthcare) using identical projections, table height and magnification. The ACh infusion was terminated if significant ischaemia or bradycardia were observed. Endothelium-independent vasomotion was finally assessed using an intracoronary bolus injection of $400 \mu \mathrm{g}$ isosorbide dinitrate. If required, a further dose of isosorbide dinitrate was administered to fully reverse any latent effects of ACh, ensuring maximal coronary artery vasodilation before a final angiogram was recorded.

\section{Quantitative coronary angiography}

Coronary angiograms were stored digitally at the Golden Jubilee National Hospital, Glasgow for subsequent analysis. An automated edge-detection software system (Centricity CA1000, GE Healthcare) was used to measure the luminal diameter of the coronary artery distal to the infusion catheter, at end diastole. The mean percentage change in luminal diameter compared to control in two distinct $5 \mathrm{~mm}$ segments was calculated for each patient following each infusion and after final nitrate injection (each analysis segment consisted of five separate measurements $1 \mathrm{~mm}$ apart). Each segment was easily identifiable by anatomical landmarks (such as side branches). Endothelial function was defined by the vasomotor response (\% change in luminal diameter) to the highest tolerated ACh infusion, compared to control. Negative changes in vessel diameter represent vasoconstriction. One patient did not receive $10^{-4} \mathrm{M}$ ACh due to recurrent atrioventricular block. All other patients received all three concentrations of ACh. All QCA analysis was performed by a single observer, blinded to all other study results.

\section{Statistical analysis}

A sample size of 25 patients was estimated to provide $80 \%$ power to detect a relationship between the independent and the dependent variables at a two-sided 0.05 significance level, if the true change in the dependent variable is 0.3 units per unit change in the independent variable. This was based on the assumption that the SD of the independent variable (oxLDL) is 0.3 and the SD of the dependent variable (endothelial function) is $0.15{ }^{16}$ All data are expressed as mean \pm SEM unless otherwise stated. Correlations were performed using the Pearson correlation coefficient. To check whether other patient characteristics influenced the relationship between EPCs and oxLDL, gender, hypertensive status and hypercholesterolaemic status were each individually added to the model. Coronary responses for each infusion were compared using analysis of variance and post hoc Dunnett's test. Statistical analysis was performed using the SPSS statistical software package V.14.0 for Windows (SPSS Inc, Chicago, Illinois, USA).

\section{RESULTS}

\section{Study participants}

In total, 33 patients consented to the study. The endothelial function study was not performed in one patient due to time constraints in the catheterisation laboratory and in another patient due to myocardial ischaemia related to deep engagement of the infusion catheter. Results for oxLDL (2 patients) and EPCs (8 patients) were not available for analysis due to machine breakdown or late sample arrival. The patient baseline characteristics are shown in table 1 . Individual patient data of plasma oxLDL concentration, EPC count and endothelial function are shown in table 2. All patients were receiving oral aspirin and clopidogrel therapy along with standard pharmacotherapy for CHD, including a high prevalence of statins.

\begin{tabular}{lc}
\hline \multicolumn{2}{l}{ Table 1 Baseline characteristics of patients } \\
\hline $\begin{array}{l}\text { Baseline characteristics of } \\
\text { patients }\end{array}$ & $\begin{array}{l}\text { Prevalence } \\
\text { ( } \mathbf{n}=33)\end{array}$ \\
\hline Age, mean \pm SD (years) & $62.4 \pm 8.36$ \\
Clinical characteristics, $\mathrm{n}(\%)$ & \\
Male & $25(75.8)$ \\
Female & $8(24.2)$ \\
Hypertension & $17(51.5)$ \\
Hypercholesterolaemia & $23(69.7)$ \\
Diabetes mellitus & $3(9.1)$ \\
Current smoker & $4(12.1)$ \\
Positive family history for CHD & $16(48.5)$ \\
Single vessel disease & $26(78.8)$ \\
Previous Ml & $9(27.3)$ \\
Previous stroke & $1(3.03)$ \\
Preserved LV function & $32(97.0)$ \\
Heart failure & $0(0.0)$ \\
Previous PCl or CABG & $3(9.1)$ \\
Drug treatment, $n$ (\%) & \\
Aspirin & $33(100.0)$ \\
Clopidogrel & $33(100.0)$ \\
ACE inhibitor & $17(51.5)$ \\
Angiotensin receptor blocker & $4(12.1)$ \\
$\beta$-blocker & $29(87.9)$ \\
Calcium channel blocker & $13(39.4)$ \\
Diuretic & $16(48.5)$ \\
Nitrate & $16(48.5)$ \\
Nicorandil & $6(18.2)$ \\
Statin & $30(90.9)$ \\
\hline CHD, coronary heart disease; PCl, percutaneous coronary \\
intervention; Ml, myocardial infarction; LV, left ventricular; CABG, \\
coronary artery bypass graft. & \\
&
\end{tabular}


Table 2 Individual patient results for oxLDL, EPCs and coronary response to $\mathrm{ACh}$

\begin{tabular}{|c|c|c|c|}
\hline Patient No & oxLDL (IU) & $\begin{array}{l}\text { EPCs } \\
\text { ( } \mu \text { L blood) }\end{array}$ & $\begin{array}{l}\% \text { Change } \\
\text { in diameter } \\
\left(10^{-4} \mathrm{M} \mathrm{ACh}\right)\end{array}$ \\
\hline 1 & 36.34 & 0.2672 & -1.6140 \\
\hline 2 & 70.33 & 0.0142 & -18.2796 \\
\hline 3 & 49.39 & 0 & -34.1583 \\
\hline 4 & 100.89 & 0.3351 & 14.1741 \\
\hline 5 & 82.65 & 0.3233 & -0.2766 \\
\hline 6 & 72.66 & 0.4110 & -14.7591 \\
\hline 7 & 65.92 & 1.1996 & -2.1605 \\
\hline 8 & 72.08 & 0.5567 & 3.3432 \\
\hline 9 & 56.38 & 0.2376 & -8.6903 \\
\hline 10 & 56.06 & ND & -6.1734 \\
\hline 11 & 44.22 & 0.4284 & -1.6225 \\
\hline 12 & 42.87 & 0.4909 & -2.3068 \\
\hline 13 & 38.77 & 0 & -37.7922 \\
\hline 14 & 53.55 & 0.2537 & -47.2260 \\
\hline 15 & 23.36 & 0.0480 & -37.0728 \\
\hline 16 & 42.52 & ND & -8.5830 \\
\hline 17 & 20.01 & ND & -17.5202 \\
\hline 18 & 36.22 & 0 & -14.9939 \\
\hline 19 & 41.86 & ND & -12.1033 \\
\hline 20 & 34.64 & 0.0976 & -19.6740 \\
\hline 21 & NS & 0.0505 & -26.7600 \\
\hline 22 & 48.35 & 0.5088 & 3.7042 \\
\hline 23 & 42.25 & ND & -3.9413 \\
\hline 24 & 36.81 & 0 & 16.5367 \\
\hline 25 & 29.26 & 0 & -19.0864 \\
\hline 26 & 29.33 & 0 & ND \\
\hline 27 & 41.92 & 0.0788 & -11.3369 \\
\hline 28 & 41.01 & 0 & -3.9623 \\
\hline 29 & 41.73 & 0.0266 & -14.1770 \\
\hline 30 & 26.31 & ND & ND \\
\hline 31 & 33.99 & ND & -1.5818 \\
\hline 32 & NS & ND & -9.2091 \\
\hline 33 & 50.65 & 0 & -39.1786 \\
\hline
\end{tabular}

ACh, acetylcholine; EPCs, endothelial progenitor cells; ND, not determined; NS, no sample; oxLDL, oxidised low-density lipoprotein.

\section{Coronary endothelial function}

The mean vessel response for each ACh infusion and isosorbide dinitrate injection are shown in figure 1 . The mean response to incremental concentrations of ACh was progressive vasoconstriction, which was significant at the highest concentration $(1.36 \pm 1.48 \%,-4.74 \pm 1.68 \%$ and $-12.27 \pm 2.74 \%$ for $10^{-6}, 10^{-5}$ and $10^{-4} \mathrm{M} \mathrm{ACh}$, respectively, $\mathrm{p}<0.001$ for $10^{-4} \mathrm{M}$ ACh vs control). The mean response to isosorbide dinitrate was $9.94 \pm 2.15 \%$, $\mathrm{p}<0.001$ versus control. There was good agreement between the two segments analysed for each patient. The mean difference in endothelial function between the two segments analysed in each patient was 0.52 (SD 14.74) percentage points. Seven of 31 patients displayed vasoconstriction in one segment and vasodilation in the other segment. In such cases, the mean response was calculated. There was a biphasic response to ACh in 5 of

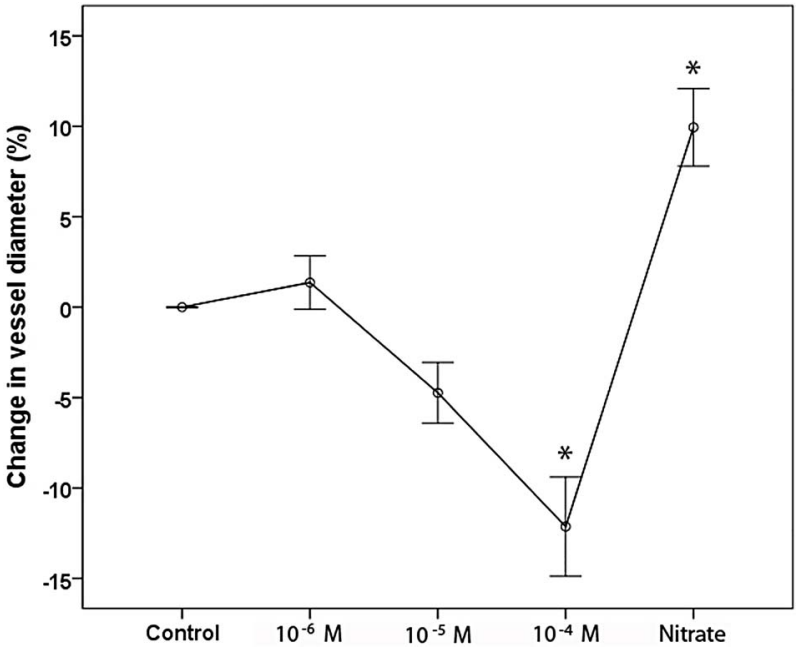

Figure 1 Mean vasomotor response to serial acetylcholine infusions and isosorbide dinitrate. There was significant vasoconstriction during the highest acetylcholine concentration whereas administration of isosorbide dinitrate caused vasodilation. $n=31 ;{ }^{*} p<0.001$ vs control.

31 patients, with a vasodilatory response at $10^{-6} \mathrm{M}$ of $\mathrm{ACh}$ ( $\geq 5 \%$ increase in vessel diameter) and vasoconstriction at $10^{-4} \mathrm{M}$ ACh ( $\geq 5 \%$ decrease in vessel diameter).

\section{Adverse events}

Transient atrioventricular block was relatively common during ACh infusion (occurring in 5 patients), especially during the highest concentration, but this was always short-lived (less than $10 \mathrm{~s}$ ) after the infusion was stopped. One patient developed transient atrial fibrillation. Clinical evidence of ischaemia was rare, occurring in only two patients. Figure 2 shows an example of severe vasoconstriction in the left anterior descending coronary artery in one patient who developed marked ischaemia during high-dose ACh infusion; this was quickly reversed with intracoronary nitrate injection. No serious or lasting complications were encountered.

\section{Correlations}

The mean plasma oxLDL concentration was $47.2 \mathrm{IU}$ and the mean number of circulating EPCs in venous blood was $0.21 \pm 0.06$ per $\mu \mathrm{L}$. There was a significant positive correlation between oxLDL and the number of circulating EPCs $(r=0.46, p=0.02)$. The individual data for these results are displayed in figure 3A. Adding hypertensive status, hypercholesterolaemic status or gender individually to the model did not affect the relationship between oxLDL and EPCs (data not shown). Diabetes mellitus and smoking were present in no greater than three patients, so these variables were not included in the analysis due to insufficient power to detect an effect. One patient had a much greater value for EPCs and to ensure the regression was not unduly influenced by this value, a sensitivity analysis was conducted by was removing this data point from the model. Following this, the 


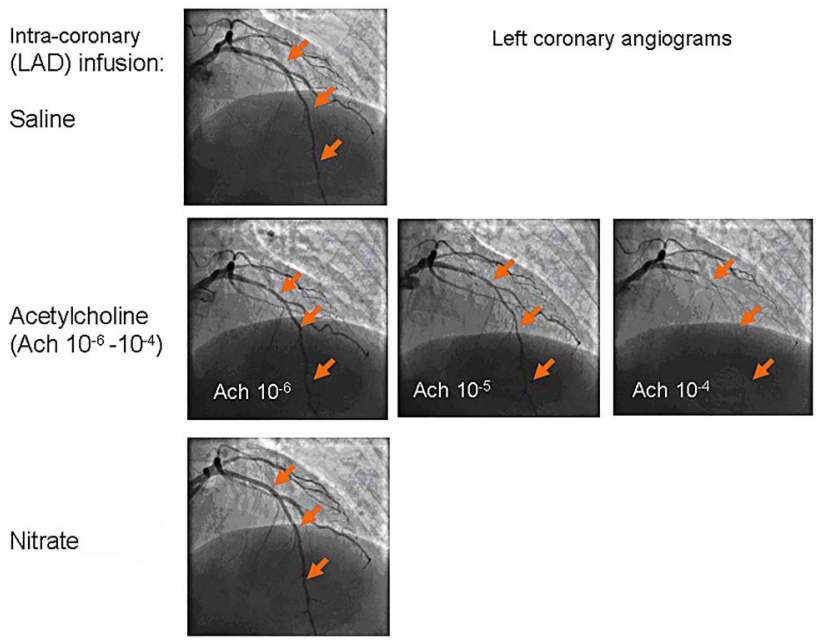

Figure 2 Angiographic example of severe coronary endothelial dysfunction. Progressive vasoconstriction of the left anterior descending artery after serial acetylcholine infusions. Isosorbide dinitrate injection caused complete reversal of vasoconstriction. LAD, left anterior descending.

correlation between oxLDL and EPCs remained significant $(\mathrm{p}=0.02)$. There was also a significant positive correlation between the number of circulating EPCs and coronary endothelial function $(\mathrm{r}=0.42, \mathrm{p}=0.04)$. This data are shown in figure 3B. Thus, patients with fewer circulating EPCs displayed more severe coronary endothelial dysfunction. Age inversely correlated with circulating EPC levels $(\mathrm{r}=-0.46, \quad \mathrm{p}=0.02)$. Given the potentially confounding effect of statins with regard to EPCs and endothelial function, an exploratory analysis was performed excluding three patients not prescribed a statin. After excluding these patients, the positive correlation between oxLDL and the number of circulating EPCs $(\mathrm{r}=0.43, \mathrm{p}=0.04)$ and EPCs and endothelial function $(\mathrm{r}=0.53, \mathrm{p}=0.01)$ remained significant. There was no significant correlation between oxLDL and coronary endothelial function $(\mathrm{r}=0.31, \mathrm{p}=0.11)$. Endothelium-independent vasodilation induced by isosorbide dinitrate was not correlated with any parameter.

\section{DISCUSSION}

This study tested coronary endothelial function in patients with stable CHD and assessed its relationship with circulating levels of EPCs and oxLDL. The major novel findings of this study were as follows: (1) There was a high prevalence of coronary endothelial dysfunction in this patient population; (2) oxLDL correlated positively with EPCs; (3) EPCs correlated positively with coronary endothelial function.

\section{OxLDL and EPCs}

The mean EPC count in the study population is broadly in agreement with previous studies. ${ }^{17}$ A novel finding was that oxLDL measured in the peripheral blood of patients with CHD was positively correlated with
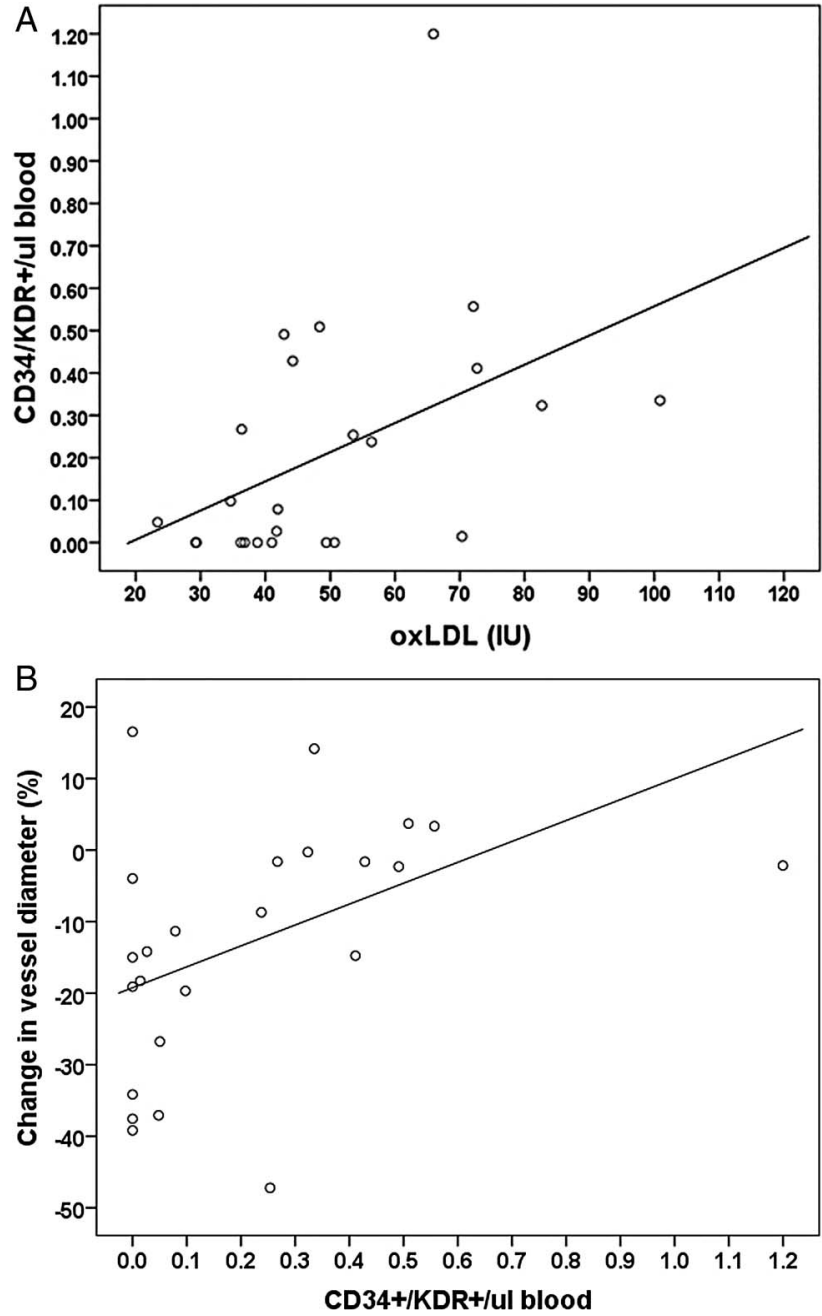

Figure 3 (A) Scatter plot of plasma oxLDL concentration and the number of circulating EPCs (CD34+/KDR+ cells). OxLDL was correlated with EPC numbers $(n=24 ; r=0.46$; $\mathrm{p}<0.05)$. (B) Scatter plot of the number of circulating EPCs (CD34+/KDR+ cells) and coronary endothelial function. Endothelial function is represented by \% change in vessel diameter during acetylcholine, compared to control. EPC numbers were correlated with endothelial function $(n=24$; $r=0.42 ; p<0.05$ ). EPCs, endothelial progenitor cells; oxLDL, oxidised low-density lipoprotein.

circulating EPC numbers. This result was contrary to much of the literature reporting in vitro toxicity of oxLDL toward EPCs. Besides the diverse methods to identify EPCs in the literature, our finding may be explained by a number of issues. In previous studies, EPC dysfunction occurred at $1-10 \mu \mathrm{g} / \mathrm{mL}$ oxLDL ${ }^{11} 12$ and EPC apoptosis (5-10\%) was induced only by $25 \mu \mathrm{g} / \mathrm{mL}$ oxLDL or higher. ${ }^{9} 1018$ Wang et al ${ }^{19}$ reported a reduction in cultured EPCs $(\sim 50 \%)$ at a concentration of $100 \mu \mathrm{g} / \mathrm{mL}$ oxLDL. However, in contrast, low concentrations $(5 \mu \mathrm{g} / \mathrm{mL})$ of oxLDL were found to have a positive effect on EPC tube formation through activation of endothelial nitric oxide synthase (eNOS) ${ }^{20}$ In our study, the mean plasma oxLDL concentration of $47.2 \mathrm{IU}$ was equivalent to $14.1 \mu \mathrm{g} / \mathrm{mL}$ (personal communication 
from manufacturer). Furthermore, studies that investigated the protective effect of statins found that oxLDL-induced EPC dysfunction and senescence were profoundly inhibited by $1 \mu \mathrm{M}$ atorvastatin, which may occur via activation of Akt. ${ }^{11}{ }^{12}$ Indeed, statin 'reloading' can be used to raise the numbers of circulating EPCs in patients undergoing PCI. ${ }^{21}$ Thus, the relatively low concentration of oxLDL in the blood and liberal use of statins in our contemporary CHD population may have protected circulating EPCs against oxLDL-induced damage. That circulating EPCs were actually increased in those with higher oxLDL concentration is a novel finding that merits further consideration and confirmatory study. We postulate that this positive correlation may be due to a host-repair response, induced by the damaging effects of increased circulating oxLDL on the vasculature; or that plasma oxLDL is a trigger (direct or indirect) for the release and mobilisation of EPCs from the bone marrow. OxLDL stimulates the release of several proinflammatory chemokines in patients with $\mathrm{CHD}^{22}$ and one or more of these factors may have been responsible for increased numbers of EPCs in the circulation. For instance, oxLDL markedly stimulates the release of interleukin (IL)-8 and growth regulated oncogene alpha (GRO- $\alpha$ ) from peripheral blood mononuclear cells and platelets in patients with CHD. ${ }^{22}$ These family of IL-8/GRO- $\alpha$ chemokines have since been shown to promote the homing of EPCs to areas of ischaemic myocardium. ${ }^{23}$ The number of circulating EPCs may also be increased in acute coronary syndromes ${ }^{424}$ and severe forms of CHD, ${ }^{5}$ which are both associated with elevated oxLDL in the blood. ${ }^{25} \mathrm{~A}$ recent study has found rapid intracoronary recruitment of EPCs in patients with ST elevation myocardial infarction, likely to represent a reparative response. ${ }^{4}$

\section{EPCs and endothelial function}

We have shown that the number of circulating EPCs in the peripheral blood of patients with stable CHD was correlated with coronary endothelial function. Hill et $a l^{26}$ previously showed in 45 individuals without cardiovascular disease that peripheral vascular function (assessed by flow-mediated brachial artery reactivity) was correlated with the number of EC colony forming units in culture, thought at the time to represent EPCs derived from peripheral blood. It has since been established that this widely cited study assessed an aspect of endothelial biology that did not reflect the number of actual EPCs present in the circulation. ${ }^{27}$ EPCs are widely believed to originate from haematopoietic stem cells, which are positive for CD34 (or the more immature marker protein $\mathrm{CD} 133$ ) and the EC antigen, KDR. These putative EPCs appear to make a valuable contribution to vessel formation. Hence, the measurement of CD34+/KDR+ cells is thought to be the most appropriate way to define circulating EPCs, ${ }^{27}$ consistent with our study. Werner et $a l^{28}$ reported a positive correlation between EPCs and coronary endothelial function and their finding has not, to the best of our knowledge, been confirmed since by others. This study in 90 patients with stable CHD measured the number of circulating EPCs in peripheral blood using flow cytometry to quantify CD34+/KDR+ and CD133+ cells. The ability of EPCs to produce endothelial colony forming units was also measured. It was found using univariate analysis that patients with low EPC number had the most severely impaired coronary endothelial function and $\mathrm{CD} 34+/ \mathrm{KDR}+$ cells were more strongly correlated with endothelial function than CD133+ cells. The failure of endothelial colony forming units to independently predict endothelial function probably relates to their dissociation with the number of circulating CD34+/KDR+ cells. ${ }^{27}$ By improving the capacity of the endothelium to be repaired after vessel injury, an obvious link between circulating EPC numbers and coronary endothelial function exists. The confirmation herein and by Werner $e t a l^{28}$ that lower numbers of EPCs are associated with impaired coronary endothelial function provides a pathological basis for the ability of EPCs to predict cardiovascular outcome. ${ }^{29}$

\section{Limitations}

The method to identify EPCs in this study using specific haematopoietic and endothelial markers, CD34 and $\mathrm{KDR}$, is consistent with contemporary guidance, ${ }^{27}$ but the most accurate way to quantify EPCs remains speculative. It may have been useful to assess EPC function, as this may have revealed a negative association with plasma oxLDL levels and provided insights into endothelial dysfunction present in patients with elevated oxidative stress. We did not measure oxLDL antibodies which may have provided an assessment of oxLDL formation over a longer time period, however, the correlation between these variables is not linear. ${ }^{30}$ Although, vasodilator drugs were withheld for $24 \mathrm{~h}$ before assessment of endothelial function, studies have shown that calcium channel blockers ${ }^{31}$ and ACE inhibitors ${ }^{32}$ can increase circulating EPCs. Thus, we cannot rule out the possibility that variable uptake of non-statin cardioprotective medications contributed to the correlations we found in our relatively small study.

\section{CONCLUSIONS}

This observational study has confirmed a high prevalence of coronary endothelial dysfunction in patients with stable CHD. Plasma oxLDL correlated with the number of circulating EPCs in the blood, possibly due to the presence of a host-repair response and the protective effects of prescribed statins. Coronary endothelial function in this population was correlated with the number of circulating EPCs, which has been confirmed by one other group. ${ }^{28}$ Future studies should aim to define the reasons for reduced numbers of EPCs in cases of severe endothelial dysfunction and identify strategies to prevent this decline. 
Acknowledgements The authors would like to thank Ms Anne McGarrity and Dr Paul Welsh (both University of Glasgow) for technical assistance with the laboratory assays and Mr Graham Conkie (Western Infirmary Pharmacy Production Unit) for supply of acetylcholine infusions. This paper is dedicated to the memory of Professor Roger M Wadsworth.

Contributors JDM, NA and JH contributed to the analysis of the data and to writing parts of the manuscript. JW, CB and KGO contributed to study design, data collection and analysis and preparation of the manuscript. SK and RMW contributed to study design, data analysis and writing and proof-reading the manuscript.

Funding This work was supported by a British Heart Foundation (BHF) Junior Research Fellowship (FS/05/096/19933) awarded to Dr J Watt.

Competing interests None declared.

Patient consent Obtained.

Provenance and peer review Not commissioned; externally peer reviewed.

Data sharing statement All collected data from the study are presented in the manuscript.

Open Access This is an Open Access article distributed in accordance with the terms of the Creative Commons Attribution (CC BY 4.0) license, which permits others to distribute, remix, adapt and build upon this work, for commercial use, provided the original work is properly cited. See: http:// creativecommons.org/licenses/by/4.0/

\section{REFERENCES}

1. Li D, Mehta JL. Oxidized LDL, a critical factor in atherogenesis. Cardiovasc Res 2005;68:353-4.

2. Takahashi T, Kalka $\mathrm{C}$, Masuda $\mathrm{H}$, et al. Ischemia- and cytokine-induced mobilization of bone marrow-derived endothelial progenitor cells for neovascularization. Nat Med 1999;5:434-8.

3. Massa M, Rosti V, Ferrario M, et al. Increased circulating hematopoietic and endothelial progenitor cells in the early phase of acute myocardial infarction. Blood 2005;105:199-206.

4. Porto I, De Maria GL, Leone AM, et al. Endothelial progenitor cells, microvascular obstruction, and left ventricular remodeling in patients with ST elevation myocardial infarction undergoing primary percutaneous coronary intervention. Am J Cardiol 2013;112:782-91.

5. Güven H, Shepherd RM, Bach RG, et al. The number of endothelial progenitor cell colonies in the blood is increased in patients with angiographically significant coronary artery disease. J Am Coll Cardiol 2006:48:1579-87.

6. Kunz GA, Liang G, Cuculi F, et al. Circulating endothelial progenitor cells predict coronary artery disease severity. Am Heart $J$ 2006:152:190-5.

7. He T, Peterson TE, Holmuhamedov EL, et al. Human endothelial progenitor cells tolerate oxidative stress due to intrinsically high expression of manganese superoxide dismutase. Arterioscler Thromb Vasc Biol 2004:24:2021-7.

8. Aicher A, Zeiher AM, Dimmeler S. Mobilizing endothelial progenitor cells. Hypertension 2005;45:321-5.

9. Ma F, Ren Q, Han Z. Effects of oxidized low-density lipoprotein on endothelial progenitor cells survival and activity mediated by lectin-like oxidized low density lipoprotein receptor. Zhongguo Yi Xue Ke Xue Yuan Xue Bao 2007;29:336-41.

10. Ma FX, Zhou B, Chen Z, et al. Oxidized low density lipoprotein impairs endothelial progenitor cells by regulation of endothelial nitric oxide synthase. J Lipid Res 2006:47:1227-37.

11. Imanishi T, Hano T, Sawamura T, et al. Oxidized low-density lipoprotein induces endothelial progenitor cell senescence, leading to cellular dysfunction. Clin Exp Pharmacol Physiol 2004;31:407-13.

12. Imanishi T, Hano T, Matsuo Y, et al. Oxidized low-density lipoprotein inhibits vascular endothelial growth factor-induced endothelial progenitor cell differentiation. Clin Exp Pharmacol Physiol 2003;30:665-70.
13. Penny WF, Ben-Yehuda O, Kuroe K, et al. Improvement of coronary artery endothelial dysfunction with lipid-lowering therapy: heterogeneity of segmental response and correlation with plasma-oxidized low density lipoprotein. J Am Coll Cardiol 2001;37:766-74.

14. Fadini GP, Losordo D, Dimmeler S. Critical reevaluation of endothelial progenitor cell phenotypes for therapeutic and diagnostic use. Circ Res 2012;110:624-37.

15. Deanfield JE, Halcox JP, Rabelink TJ. Endothelial function and dysfunction: testing and clinical relevance. Circulation 2007;115:1285-95.

16. Desk R, Williams L, Health K, et al. Effect of Nifedipine and Cerivastatin on Coronary Endothelial Function in Patients With Coronary Artery Disease: The ENCORE I Study (Evaluation of Nifedipine and Cerivastatin On Recovery of coronary Endothelial function). Circulation 2003;107:422-8.

17. van lerssel SH, Van Craenenbroeck EM, Hoymans VY, et al. Endothelium dependent vasomotion and in vitro markers of endothelial repair in patients with severe sepsis: an observational study. PLOS ONE 2013;8:e69499.

18. Ji KT, Qian L, Nan JL, et al. Ox-LDL induces dysfunction of endothelial progenitor cells via activation of NF-kB. Biomed Res Int 2015;2015:175291.

19. Wang X, Chen J, Tao Q, et al. Effects of ox-LDL on number and activity of circulating endothelial progenitor cells. Drug Chemical Toxicol 2004;27:243-55.

20. Lin FY, Tsao NW, Shih CM, et al. The biphasic effects of oxidized-low density lipoprotein on the vasculogenic function of endothelial progenitor cells. PLOS ONE 2015;10:e0123971.

21. Ye H, He F, Fei X, et al. High-dose atorvastatin reloading before percutaneous coronary intervention increased circulating endothelial progenitor cells and reduced inflammatory cytokine expression during the perioperative period. $J$ Cardiovasc Pharmacol Ther 2014:19:290-5.

22. Holm T, Damås JK, Holven $\mathrm{K}$, et al. CXC-chemokines in coronary artery disease: possible pathogenic role of interactions between oxidized low-density lipoprotein, platelets and peripheral blood mononuclear cells. J Thromb Haemost 2003;1: 257-62.

23. Kocher AA, Schuster MD, Bonaros N, et al. Myocardial homing and neovascularization by human bone marrow angioblasts is regulated by IL-8/Gro CXC chemokines. J Mol Cell Cardiol 2006;40:455-64.

24. George J, Goldstein E, Abashidze S, et al. Circulating endothelial progenitor cells in patients with unstable angina: association with systemic inflammation. Eur Heart $J$ 2004;25:1003-8.

25. Ehara S, Ueda M, Naruko T, et al. Pathophysiological role of oxidized low-density lipoprotein in plaque instability in coronary artery diseases. J Diabetes Complications 2002;16:60-4

26. Hill JM, Zalos G, Halcox JP, et al. Circulating endothelial progenitor cells, vascular function, and cardiovascular risk. $N$ Engl $J$ Med 2003;348:593-600.

27. George J, Shmilovich H, Deutsch V, et al. Comparative analysis of methods for assessment of circulating endothelial progenitor cells. Tissue Eng 2006;12:331-5.

28. Werner N, Wassmann $\mathrm{S}$, Ahlers $\mathrm{P}$, et al. Endothelial progenitor cells correlate with endothelial function in patients with coronary artery disease. Basic Res Cardiol 2007;102:565-71.

29. Schmidt-Lucke C, Rössig L, Fichtlscherer S, et al. Reduced number of circulating endothelial progenitor cells predicts future cardiovascular events: proof of concept for the clinical importance of endogenous vascular repair. Circulation 2005;111:2981-7.

30. Wang $\mathrm{Y}$, Fang $\mathrm{X}$, Wang $\mathrm{S}$, et al. Relation between plasma oxLDL antibodies and oxLDL in the circulation. Inflammation 2007; $30: 7-13$

31. de Ciuceis C, Pilu A, Rizzoni D, et al. Effect of antihypertensive treatment on circulating endothelial progenitor cells in patients with mild essential hypertension. Blood Press 2011;20:77-83.

32. Cacciatore F, Bruzzese G, Vitale DF, et al. Effects of ACE inhibition on circulating endothelial progenitor cells, vascular damage, and oxidative stress in hypertensive patients. Eur J Clin Pharmacol 2011;67:877-83. 